\title{
Evaluation of Continuous Direction Encoding with Tactile Belts
}

\author{
Martin Pielot ${ }^{1}$, Niels Henze ${ }^{1}$, Wilko Heuten ${ }^{1}$, Susanne Boll ${ }^{2}$ \\ ${ }^{1}$ OFFIS Institute for Information Technology, Germany, \\ ${ }^{2}$ University of Oldenburg, Germany, \\ \{pielot, henze, heuten\}@offis.de, \\ susanne.boll@uni-oldenburg.de
}

\begin{abstract}
Tactile displays consisting of tactors located around the user's waist are a proven means for displaying directions in the horizontal plane. These displays use the body location of tactors to express directions. In current implementations the number of directions that can be expressed is limited to the number of tactors. However, the required number of tactors might not be available or their configuration requires too much effort. This paper describes the design and the evaluation of a presentation method that allows displaying direction between tactors by interpolated their intensity. We compare this method with the prevalent one by letting participants determine directions and having them navigate along tactile waypoints in a virtual environment. The interpolated direction presentation significantly improved the accuracy of perceived directions. Discrete direction presentation, however, proved to be better suited for waypoint navigation and was found easier to process.
\end{abstract}

Key words: multimodal user interfaces, tactile displays, direction presentation, interpolation, orientation and navigation

\section{Introduction}

Maps and route descriptions are well established means for orienting in an unfamiliar area, keeping on track of a route, or finding points of interests (POIs). Most widely used tools for this are maps, either printed or digitally integrated in car navigation systems or mobile phones. These tools rely on the visual sense to be interpreted. In car navigation systems the visual display is complemented by a speech output which gives us directions to keep on track. However, both visual and auditory feedback might not be the most suitable ones to support a person's orientation and navigation while walking or driving. The visual display needs visual attention and is competing with the attention we need for watching and observing our surroundings. The auditory display can be perceived via speakers or earphones but can also be experienced as being annoying, obtrusive, and interfering with other tasks such as driving or talking to a friend.

In our research, we explored tactile sensation as a modality to present information for orientation and navigation. The driving argument behind this is that 
tactile sensation can be perceived in a fashion that it is not obtrusive to the current user task and also can be perceived in discretion without the environment noticing it - as we know this from mobile phones that rest in vibration mode in our pockets. Tactile displays generally "appeal to the cutaneous senses by skin indentation, vibration, skin stretch and electrical stimulation." [2]. Addressing the cutaneous sense, we developed and evaluated a tactile display that uses tactile transducers (tactors) worn around the waist. Their vibration can be sensed at different intensity levels and rhythms at the spot where they are attached to the skin. Using spatially distributed tactors each tactors' location can be used as an additional output parameter. If spatially distributed tactors are used the tactile output at a specific location on the body can be connected to specific information.

Different groups $[10,6,13,5]$ as well as ourselves [4] have shown that such tactile belts are a promising approach to provide directions in the horizontal plane. They used the tactors on the display to indicate a direction such as cardinal directions or the direction of waypoints. One drawback of the existing systems is, however, that the direction information is realized as a discrete presentation and each tactor conveys exactly one direction which results in either a high number of tactors or very coarse direction information.

In this paper, we present an evaluation of continuous direction presentation with a tactile belt. The continuous direction is encoded by intensity interpolation of adjacent tactors. Our evaluation bases on our tactile belt in which six tactors are distributed equally around a user's waist. With two experiments we compare interpolated versus discrete tactile information presentation. The study provides evidence that interpolated direction presentation leads to a more accurate perception of the direction, while the discrete direction presentation was easier to perceive and process for waypoint navigation. The two major advantages of the interpolated direction presentation is that the accuracy of the perceived direction information increases. In addition, the system design can be parameterized such that existing tactile belts with varying numbers of tactors can now be re-programmed rather than re-engineered.

\section{Related Work}

Previous work has shown the feasibility of tactile displays for presenting directions by mapping them to body locations [1]. Many application scenarios have been suggested, like maintaining spatial awareness, waypoint navigation, and displaying the location of objects such as POIs.

Tan and Pentland [9] used a tactile belt for displaying cardinal directions to the user. This belt consisted of several tactors worn around the user's waist and a compass. The system always activates the tactor that points most closely north. This kind of perception was evaluated by Nagel et al. [6]. For six weeks, four participants wore a belt which displayed north. Afterwards, a significant difference in a targeting task was observed between the experimental and the control group. Van Erp et al. [12] evaluated displaying directions for counteract- 
ing spatial disorientation. Participants were rotated around the yaw axis using a swivel chair for 24 seconds. Afterwards, they had to compensate a quasi random angular velocity disturbance generated by the chair. Van Erp et al. showed that using a tactile display helps to recover spatial orientation.

Van Erp et al. [13] showed that pedestrians are able to follow a route consisting of waypoints guided by a tactile belt only. However, they observed that their participants were walking zigzag towards the waypoints. They argue that the limitation of tactors resulted in a too inaccurate direction presentation. Tsukada and Yasumura [10] additionally found that reducing the length of vibration pulses has a negative effect on the perceptibility of directions. In our recent work [4] we could show that pedestrians can be guided close to an invisible route with a tactile belt when placing the waypoints very close to each other.

Lindeman et al. [5] proved the applicability of tactile belts for displaying stationary POIs. They evaluated user's performance in a building clearing task, where a tactile belt helped them to avoid stepping into dangerous areas by displaying hazardous spots. In our previous work we proposed a system for keeping groups together in crowded environments [7]. A tactile belt is used to display the location of the group's individuals. Rupert [8] investigated displaying pilots the location of objects around them in 3D space by using a tactile display consisting of 128 tactors worn around the whole torso.

\section{Direction Presentation with a Tactile Belt}

In our previous work, we developed a belt type tactile display with six tactors [4]. The belt consists of flexible fabrics and is worn around the hip. Six vibration motors serving as tactors are sewn into the belt. The tactors are composed of an unbalanced mass on a rotating axis and can produce vibrations of high frequencies. They are equally distributed leading to a distance of about $60^{\circ}$ between two adjacent tactors (see illustration in Figure 1).

The hardware design of our belt is basically comparable to the systems we presented in the related work section. In these systems, direction presentation using tactile belts typically follows a concept that we call "discrete direction presentation". Directions are expressed by modifying the body location of the tactile cue, where directions are mapped to body location. A tactor is activated if the corresponding direction should be displayed to the user. As the number of tactors is limited on the belt, a whole range of directions must be mapped to be displayed by one single tactor. Hence, each tactor is responsible for displaying a range of directions as illustrated in Figure 2.

This presentation method leads to an inherent inaccuracy. Taking our belt as example, each tactor is mapped to a range of directions with a size of $60^{\circ}$. Thus, the deviation between actual and expressed directions lies between $0^{\circ}$ $30^{\circ}$. This results into an average deviation of $15^{\circ}$, assuming evenly distributed directions being displayed. One solution to this problem is to alter the mapping between directions and body location considering the application scenario. For waypoint navigation, one extra tactor could indicate being on route, while the 

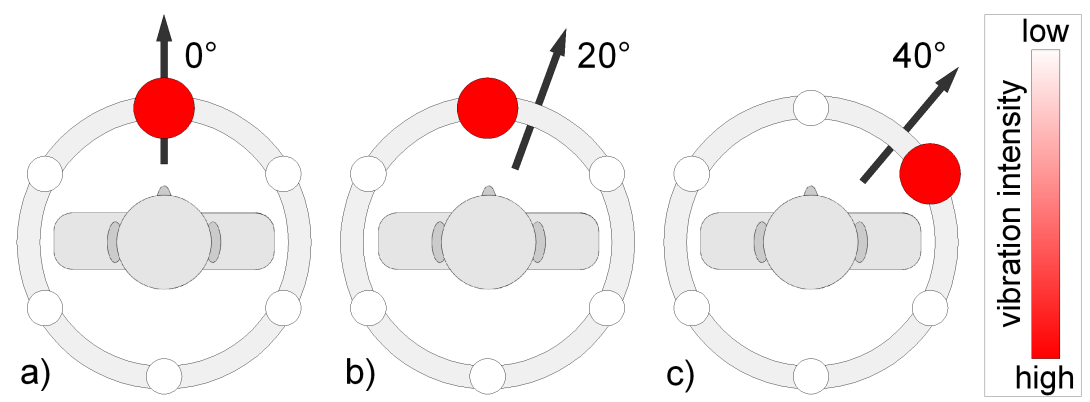

Fig. 1. Sketch of a belt with six tactors displaying three direction $\left(0^{\circ}, 20^{\circ}\right.$, and $\left.40^{\circ}\right)$ using a discrete presentation technique.

others are used to display the direction of the subsequent waypoint. However, this is not suitable for other use-cases such as displaying the location of POIs that could be located all around the user. Therefore a solution would be adding tactors to gain higher accuracy. We propose an alternative approach for gaining higher accuracy which makes best use of number of available tactors by using the vibration intensity as additional parameter for encoding directions.

Combining the parameters body location and intensity we propose a presentation method that displays directions using interpolation. This encoding exploits the effect of apparent location where a single perceived stimulus is induced by two stimuli at different locations. According to van Erp [11] the perceived location depends on the relative magnitude of the two stimuli. Directions between the exact angles of two adjacent tactors are encoded through different intensity levels of these tactors. The intensity levels are determined by a linear function depending on the displayed angle. If two tactors are $60^{\circ}$ apart, and an angle is displayed $20^{\circ}$ away from one tactor (see Figure 2), the intensity of the closer tactor is two-third, and the intensity of the other tactor is one-third of the maximum intensity. We proposed this idea in [3] for a display with three tactors. In the following this method will be called interpolated presentation. This method allows making existing devices more accurate and flexible without the need for physical re-engineering. Consequently, this opens up new options for flexible software configuration of the belt's output.

\section{Evaluation}

We conducted two experiments to compare interpolated and discrete presentation. In the first experiment we asked participants to determine directions. In the second experiment participants had to navigate along tactile waypoints in a virtual environment. We expected people to perceive directions more accurate using the interpolated presentation. We also assumed that the interpolated presentation is less obtrusive, since when the displayed direction changes slowly, no sudden jumps of the tactile feedback from one tactor to the other occur. On 

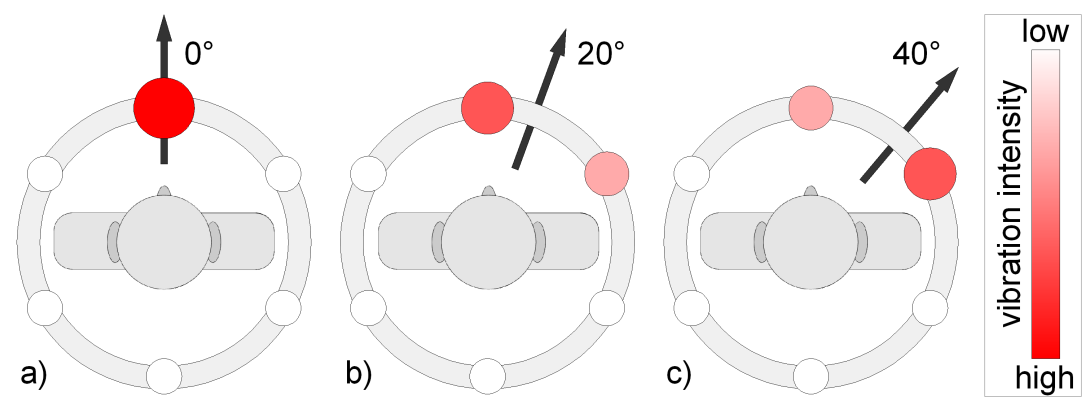

Fig. 2. Sketch of a belt with six tactors displaying three direction $\left(0^{\circ}, 20^{\circ}\right.$, and $\left.40^{\circ}\right)$ using a interpolating presentation technique.

the other hand, we supposed that discrete feedback is easier to interpret for untrained users, since there is less information to process. 16 participants with an age of 20-34 $\left(\mathrm{M}^{1}=25.73, \mathrm{SE}^{2}=1.05\right)$ took part in the evaluation. 15 of them were male and eight had previous experience with our tactile belt. We afterwards handed out questionnaires to ask for the participants' subjective impressions. The experiments and the questionnaire are described in the following.

\subsection{Accuracy of Direction Perception}

The aim of the first experiment was to test the assumptions that interpolated presentation is more accurate but more difficult to process. We therefore let the participants determine a number of directions displayed with both presentation methods and compared the reaction time of the users as well as the accuracy of the perceived directions.

Method Discrete presentation served as control condition and interpolated presentation as experimental condition. Every participant contributed to both groups. To rule out systematic sequence effects, we randomly assigned which display method was used first. We measured the average deviation of the determined directions for comparing the accuracy of the presentation methods. Additionally we recorded the reaction time as an indicator for the difficulty.

The tactile belt was connected to a desktop computer. An application running on the computer was used to display directions via the tactile belt. A circle on the screen enabled the participants to select the perceived direction using a mouse (see Figure 3). No other visual cues were given except a line marking front.

Prior to the experiments 32 random directions between $0^{\circ}$ and $359^{\circ}$ were generated. We displayed the same directions to every participant for comparability purposes. At the beginning of each experiment session, each participant

\footnotetext{
1 Mean

${ }^{2}$ Standard error
} 


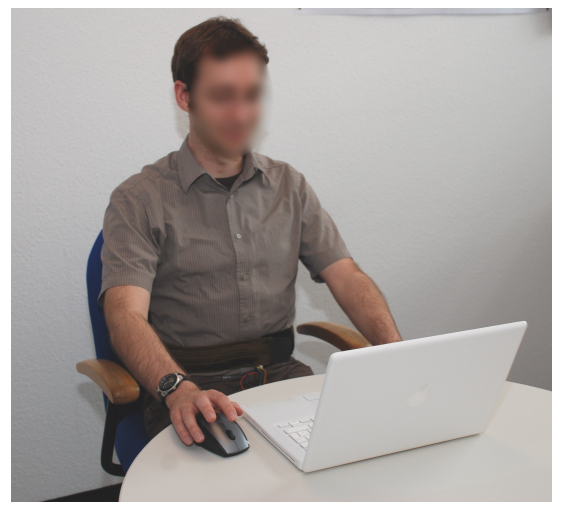

(a)

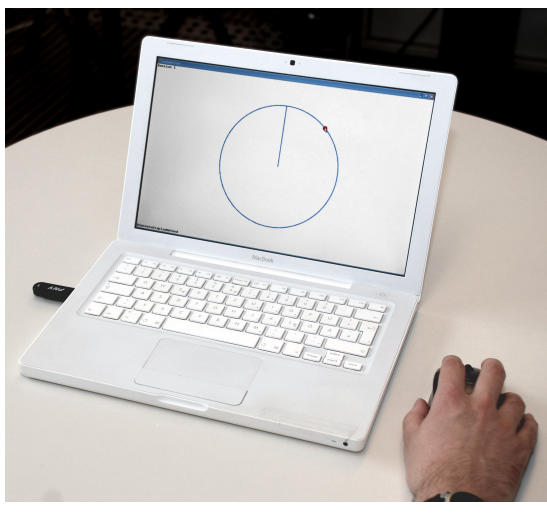

(b)

Fig. 3. (a) Participant during the evaluation wearing the tactile belt. (b) Application to specify the perceived direction.

was introduced to both display methods theoretically. Then, the application demonstrated both presentation methods by displaying a virtual point running clockwise around the participant. When the participants had familiarized themselves with the presentation methods, they were informed which method was used first to display directions. The application then presented the 32 directions in random order. Each direction was presented until the participant responded to the system by indicating the perceived direction. Afterwards, all directions were presented again in another random order using the other presentation method.

Results The mean deviation of directions given by the participants was significantly lower with the interpolated presentation $\left(16.83^{\circ}\right.$, SE 0.74$)$ compared to the discrete presentation $\left(19.43^{\circ}\right.$, SE 0.97$)$. In contrast, the reaction time was significantly higher for the interpolated presentation (4.42s, SE 0.44) than for the discrete presentation (3.23s, SE 0.26). Statistically, interpolation had a medium effect on improving the accuracy $(\mathrm{t}(14)=2.93, \mathrm{p}<.01, \mathrm{r}=.49)$ and a a high effect on prolonging the reaction time $(\mathrm{t}(14)=-4.54, \mathrm{p}<.001, \mathrm{r}=.84)$. Comparing the performance of participants that had previous experience with the tactile belt to those who had none did not reveal any significant effect.

\subsection{Waypoint navigation in virtual environments}

The goal of the second experiment was to test, how interpolated presentation performs in an exemplary task like waypoint navigation. We asked the participants to walk along a route in a virtual 3D environment. We measured and compared the time the participants needed to complete the route with each presentation method. We made no assumption about which presentation method would allow faster completion of the route. On the one hand, we expected that 
more accurate feedback would result in the ability to follow the ideal route more closely, but on the other hand, the expected higher difficulty of processing the feedback could nullify or invert this effect.

Method The participants explored a virtual area from the first person view. They had to follow a route marked by tactile waypoints. To avoid participants using landmarks as orientation help, we did not include any visible objects except the ground. Repeating, chessboard-like textures on the ground allowed users to detect their movement visually. The application allowed the user to continuously turn left and right and move forward by holding the respective arrow keys pressed. A waypoint was reached, when the participant came closer than 40 Units. All routes consisted of 5 sections with 3 waypoints each. The location of the waypoints was generated randomly with the constraint that each section had to be the same length (1000 Unit) and the same angle sum $\left(287^{\circ}\right)$. Thus, the ideal travel time for each route was the same, making the participants' performance comparable. Figure 4 shows the virtual environment and a user navigating along the route.
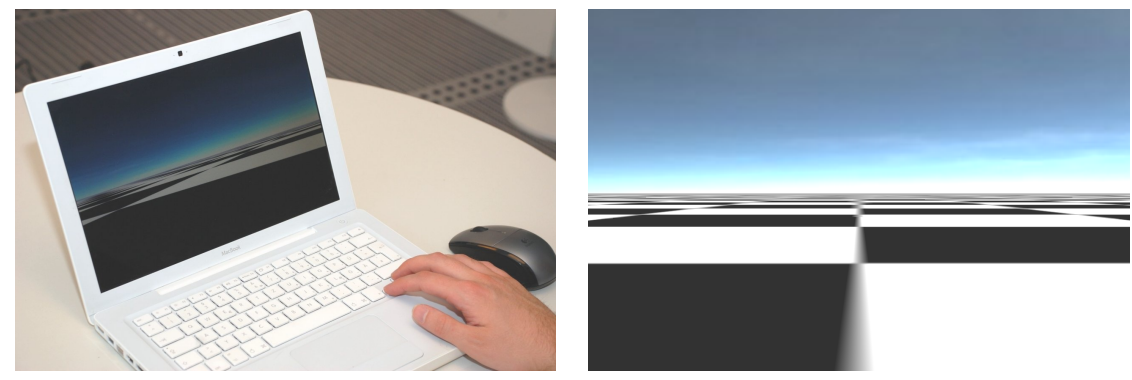

Fig. 4. Visual 3D presentation provided to the participants.

Each participant completed two routes, one using discrete and the other using interpolated presentation. The presentation method that was used first was randomly assigned to avoid systematic sequence effects. We measured the time the participants needed to complete each route to assess the participant's performance. We chose completion time as dependent variable, since besides distance covered by the participant it also takes situations into account where the user stands and turns around. Since movement speed and turning speed are both constant, this measure is an indicator for how accurate the participants stayed on the track as long as they do not halt without any reason.

Every participant had previously attended the first study. They were all introduced to the tactile belt and both presentation methods. The participants were told to complete the route as fast as possible and not to stop unnecessarily. Only, if the next waypoint was not in front of them, they should halt while 
turning. This should avoid the users walking away from waypoint, which would artificially degrade the measured performance. The experiment started, when the participants reached the first waypoint. Once they had completed the first route, the presentation method was changed and the second route started.

Results We had no training session. Instead, we dedicated the first two sections of the route as training part. For the results we therefore only take the completion time of the last three sections of each route into account. We excluded two participant's results, because they experienced technical failures and thus struggled finding the waypoints. The completion time was significantly shorter with discrete presentation $(65.59 \mathrm{~s}$, SE 3.05) compared to interpolated presentation (72.66s, SE 3.81). The presentation method had a large effect on the completion time $(\mathrm{t}(12)=-2.67, \mathrm{p}<.05, \mathrm{r}=.72)$. In general, participants walked straight towards the subsequent waypoint. We could not confirm the zigzag movement observed by van Erp et al. [13]. The most time got lost, if participants missed a waypoint and consequently had to turn around. In a few cases we observed participants circling around a waypoint, missing it several times. This happened with both presentation methods.

\subsection{Self reports and observations}

After both experiments we handed out questionnaires, assessing the subjective impression of the participants about both display methods. The participants were asked to rate for each presentation method, how obtrusive it felt, how certain they were about the correctness of the directions they determined in the first task, how easy the determination directions in the first task was, and how easy it was to follow the route in the second task. Every aspect was rated on a five point Likert scale, ranging from zero (obtrusive/uncertain/difficult) to four (unobtrusive/certain/easy).

The ratings showed that during the first experiment the participants found discrete presentation $(\mathrm{Mdn}=3$ ) significantly easier to interpret than interpolated presentation $(\mathrm{Mdn}=2)$. Discrete presentation had a medium effect on the difficulty (Wilcoxon signed-rank test: $T=82, p<.05, r=0.38$ ). There were no significant differences found between the presentation methods regarding the other three rating categories. Both presentation methods were found slightly comfortable in average $(\mathrm{Mdn}=3)$. Independent of the method, participants were slightly certain that the directions they had given were correct $(\mathrm{Mdn}=3)$, and found it slightly easy to follow the invisible route in the second experiment $(\mathrm{Mdn}=3)$.

During the evaluation several participants spontaneously mentioned that they were getting less sensitive to the vibration pulses. Consequently they found it harder to determine directions. In the first experiment, there were three cases where participants touched the belt to localize the tactile cues, since they were not able to determine their origin. Two participants even experienced a case, where they did not perceive the vibration anymore. After the first experiment, some participants indicated that determining directions was a very exhausting task and that especially interpolation is more difficult to interpret. 


\subsection{Discussion}

The first experiment confirmed our assumption that the interpolated presentation method allows presenting directions more accurately compared to the discrete presentation method. This benefit came at the expense of slower reaction time. Despite the better accuracy, the second experiment showed that a route consisting of haptically presented waypoints is completed faster with discrete presentation. The questionnaires revealed no differences between the presentation methods except that the participants found it easier to interpret directions with discrete presentation in the first experiment.

The average accuracy of interpolated presentation found in the first experiment was similar to the accuracy we found with the same presentation method during earlier studies [4]. The accuracy of discrete direction presentation was worse than anticipated. As explained in section 3 we expected an inherent inaccuracy of $15^{\circ}$ for the discrete presentation. The actual result was $19.43^{\circ}$. The difference between those values can serve as an indicator for variance decreasing the accuracy, such as the difficulty of mapping the physical experience to a visual circle or cases when participants were not able to determine which vibrator was activated.

The participants' impression that interpolated feedback is more difficult to process is backed up by the in average longer reaction time measured in the first experiment. Previous work showed that processing tactile feedback can be successfully trained [6]. However, we could not find a significant difference caused by previous experience with the tactile belt. We therefore suggest that if processing interpolated feedback can be trained, it requires more practice.

The longer reaction time might also have been a reason for discrete presentation resulting into faster completion of the route, in the second experiment. We suspect that slight changes were noticed later due to the longer processing time of interpolated feedback and due to vibration insensitiveness, since the feedback was almost always perceived from the front.

Our assumption that interpolated presentation is perceived as less obtrusive could not be confirmed. However, due to the nature of the conducted experiments those abrupt changes in the output were perceived using both presentation techniques. In the real world the relative direction of objects, such as waypoints and POIs, do not change abruptly but continuously. Sudden changes in the display's tactile output would not occur with interpolated presentation. Thus, we assume to obtain different results for real world tasks.

\section{Conclusion and Future Work}

In this paper we presented a presentation method for tactile belts that displays any direction in the horizontal plane using six vibrators only. The developed presentation method displays a direction by interpolating the intensity of two adjacent tactors. In two experiments this presentation method was compared to the discrete presentation method used by other belt type tactile displays. The 
experiments showed that interpolated presentation is more accurate than discrete presentation for the developed tactile belt with six vibrators. However, it was also found that interpolated presentation is more exhausting and takes longer to be interpreted. Despite the increased accuracy, interpolated presentation performed worse in a waypoint finding task compared to discrete presentation.

In our future work, we will use the data of the presented study to refine the interpolation. A promising approach is to replace the used linear interpolation function by a more sophisticated one. The extinction of the tactile sensation also has to be considered. Additionally, we plan to experiment with encoding additional information through other parameters of the tactile output. In particular, we are interested in using tactile displays for the presentation of localized objects, such as POIs, landmarks, or persons.

\section{References}

1. S. Brewster and L. M. Brown. Tactons: structured tactile messages for non-visual information display. In Proc. of the Australasian conference on user interface, 2004.

2. S. Brewster, S. Wall, L. Brown, and E. Hoggan. The Engineering Handbook on Smart Technology for Aging, Disability and Independence, chapter Tactile Displays. John Wiley \& Sons, Computer Engineering Series, 2008.

3. N. Henze, W. Heuten, and S. Boll. Non-intrusive somatosensory navigation support for blind pedestrians. In Proc. of Eurohaptics 2006, 2006.

4. W. Heuten, N. Henze, S. Boll, and M. Pielot. Tactile wayfinder: A non-visual support system for wayfinding. In Proc. of NordiCHI, 2008.

5. R. W. Lindeman, J. L. Sibert, E. Mendez-Mendez, S. Patil, and D. Phifer. Effectiveness of directional vibrotactile cuing on a building-clearing task. In Proc. of the conference on human factors in computing systems, 2005.

6. S. Nagel, C. Carl, T. Kringe, R. Martin, and P. Konig. Beyond sensory substitutionLearning the sixth sense. Journal of Neural Engineering, 2, 2005.

7. M. Pielot, N. Henze, and S. Boll. FriendSense: Sensing your Social Net at Night. In Workshop Night and Darkness: Interaction after Dark in conjunction with CHI 2008, 2008.

8. A. Rupert. Tactile situation awareness system: Proprioceptive protheses for sensory deficiencies. Aviation, Space and Environmental Medicine, 71:92-99, 2006.

9. H. Z. Tan and A. Pentland. Tactual displays for wearable computing. In Proc. of the International Symposium on Wearable Computers, 1997.

10. K. Tsukada and M. Yasumura. Activebelt: Belt-type wearable tactile display for directional navigation. In Proc. of the Conference on Ubiquitous Computing, 2004.

11. J. B. F. Van Erp. Guidelines for the use of vibro-tactile displays in human computer interaction. In Proc. of Eurohaptics 2002, 2002.

12. J. B. F. Van Erp, E. L. Groen, and J. E. Bos. A tactile cockpit instrument supports the control of self-motion during spatial disorientation. Human Factors: The Journal of the Human Factors and Ergonomics Society, 48:219-228, 2006.

13. J. B. F. Van Erp, H. A. H. C. Van Veen, C. Jansen, and T. Dobbins. Waypoint navigation with a vibrotactile waist belt. ACM Transactions on Applied Perception, $2: 106-117,2005$. 